\title{
Subject Visits Domain
}

National Cancer Institute

\section{Source}

National Cancer Institute. Subject Visits Domain. NCI Thesaurus. Code C49617.

A subject domain utilized for the submission of information encompassing and representing data, vocabulary or records related to subject visit. 